Journal of Innovative Optical Health Sciences

Vol. 9, No. 6 (2016) 1550039 (11 pages)

(C) The Author(s)

DOI: $10.1142 / \mathrm{S} 179354581550039 \mathrm{X}$

\title{
An online identity authentication method for blood smear
}

\author{
Xiaozhen Feng, Yiping Cao*, Kuang Peng and Cheng Chen \\ Department of Optical Electronics \\ Sichuan University \\ Chengdu, Sichuan 610064, P. R. China \\ *ypcao@scu.edu.cn
}

Received 29 January 2015

Accepted 17 April 2015

Published 20 May 2015

\begin{abstract}
Blood smear test is the basic method of blood cytology and is also a standard medical test that can help diagnose various conditions and diseases. Morphological examination is the gold standard to determine pathological changes in blood cell morphology. In the biology and medicine automation trend, blood smears' automated management and analysis is very necessary. An online blood smear automatic microscopic image detection system has been constructed. It includes an online blood smear automatic producing part and a blood smear automatic microscopic image detection part. Online identity authentication is at the core of the system. The identifiers printed online always present dot matrix digit code (DMDC) whose stroke is not continuous. Considering the particularities of DMDC and the complexities of online application environment, an online identity authentication method for blood smear with heterological theory is proposed. By synthesizing the certain regional features according to the heterological theory, high identification accuracy and high speed have been guaranteed with few features required. In the experiment, the sufficient correct matches between the tube barcode and the identification result verified its feasibility and validity.
\end{abstract}

Keywords: Blood smear; digit identification; identity authentication; feature identification; blood smear detection; microscopic imaging.

\section{Introduction}

Morphological examination is the gold standard to determine pathological changes in blood cell morphology. Blood smear is the basic method of blood cytology, and it has been applied widely,$^{1-3}$ especially as it adds great value to the diagnosis of various blood diseases. ${ }^{4,5}$ Blood smears automated management and analysis is necessary in biology and medicine automation trend. Identity authentication for blood smear has been of great concern, for it is the premise of automatic management. The identifier is the only connection between the smear and

*Corresponding author.

This is an Open Access article published by World Scientific Publishing Company. It is distributed under the terms of the Creative Commons Attribution 4.0 (CC-BY) License. Further distribution of this work is permitted, provided the original work is properly cited. 


\section{Feng et al.}

the patient information, so the accuracy of the identity authentication for blood smear is pretty important. In the blood smear making process, the bar code on the tube should be transformed to be printed on the planar smear as the identifier online. Owing to the online printing limitation, the free movement of the blood smear may cause printed bar code deformed, fuzzy, or even parts of it printed outside the smear, which will severely reduce the reliability of barcode. What is more, the barcode printing device costs too much space, which cannot be admitted by our online blood smear automatic microscopic image detection system. Besides that, as for online full-automatic system, the first-reading rate is required to be $100 \%$. We have tested the barcode option with several high-quality barcode devices, such as Honeywell, Motorola and so on. We found that the first-reading rates based on every 200 samples are all below 99\%, which caused wrong correspondence between patients and smears. Thus, we get the conclusion that bar code is no longer applicable at this circumstance and the digit code takes the place.

In the automatic management system, stylus printer is widely used for its unique advantages of pocket-size and easy control with single-chip microcomputer. And the first-reading rate with it can be adapted to be $100 \%$, which is much important for online full-automatic system. The printed digit code, presenting dot matrix digit code (DMDC) whose stroke is not continuous also takes the advantages of good readability without any additional reading device and small area requirement for printing. Nevertheless, the existing feature types and extraction techniques are mostly for the digit code with continuous stroke. ${ }^{6-9}$ The most popular feature is the direction feature, which can be measured from skeleton, ${ }^{10}$ chaincode, ${ }^{11}$ or gradient. ${ }^{12}$ And in addition some complementary features such as structural and concavity feature, ${ }^{13,14}$ profile shape feature, ${ }^{15}$ and curvature feature ${ }^{16}$ yield high performance for continuous character identification. In DMDC, there are sufficient intervals among the dots and there must be gray unbalancedness of these dots caused by the stylus, or even some of the dots will be faded out and blur the structure of DMDC. Besides, if some blots as similar with the printed dots exist in the identified region and cannot be filtered out, the structure of DMDC may be confused. The DMDC is much different to those digit codes with continuous strokes in image segmentation, stroke judging and identification. The requirement is hard to meet for the above digit code identification methods which perform well with continuous stroke digit code.

In addition, although the off-line digit code identification technology is relatively mature, ${ }^{17-19}$ the online digit code identification has been an imperative demanding issue in automation system due to the uncertainty of the working environments such as image distortion, gray inequality problem which is caused by the variation of light intensity, uneven illumination, different shooting angles and the random position of the code resulted by uncertain moments.

Considering the particularities of DMDC and the complexities of online application environment, we proposed an online identity authentication method for blood smear with heterological theory. By synthesizing certain regional features according to the heterological theory, high identification accuracy and high speed have been guaranteed with few features required. And this method has been successfully applied in the patient identification of online blood smear automatic microscopic image detection which confirms its effectiveness.

\section{Online Blood Smear Code Image Preprocessing Analysis}

An online blood smear automatic microscopic image detection system is constructed, shown in Fig. 1. It includes an online blood smear automatic producing part and a blood smear automatic microscopic image detection part shown in Figs. 1(a) and 1(c), respectively. Figure 1(b) is the blood smear produced online. And Fig. 1(d) is the blood microscopic image captured online, which will be used for analysis. Online blood smear automatic microscopic image detection system is supposed to be efficient with a large amount of blood smears. There is a DMDC on each blood smear as the identifier contacting to the patient. The blood smears with DMDC is shown in Fig. 1(b). As a part of the detection management system, our task is to collect the pictures of the identifier online and transform the images into computer data without any deviation, or it will lead to unpredictable but serious consequences. High accuracy and speed of the identity authentication is crucial to the online blood smear automatic microscopic image detection. An online blood smear automatic microscopic image 


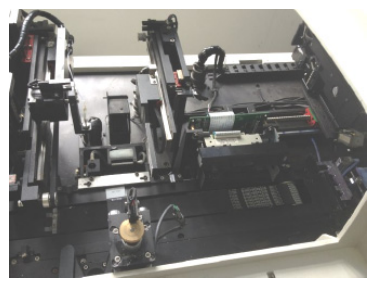

(a)

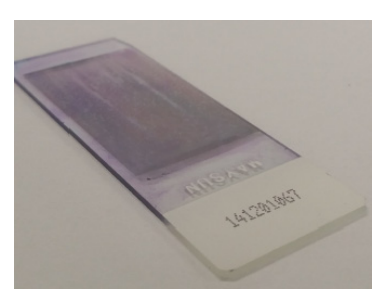

(b)

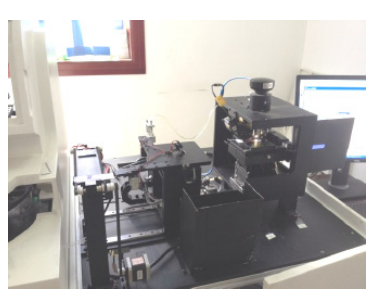

(c)

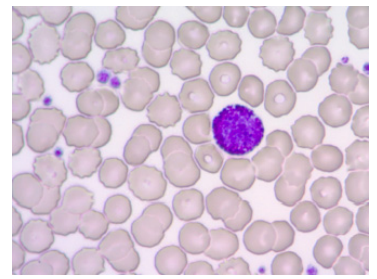

(d)

Fig. 1. The online blood smear automatic microscopic image detection construction. (a) Blood smear producing part, (b) blood smears, (c) image capturing part and (d) microscopic image captured.

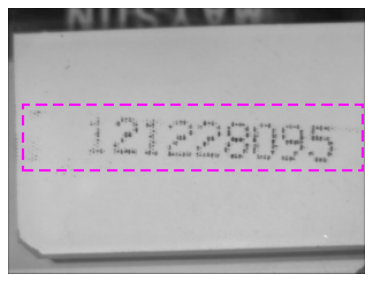

(a)

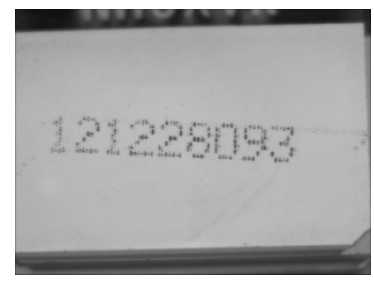

(b)

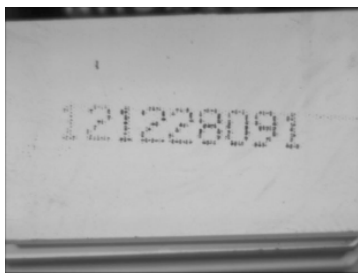

(c)

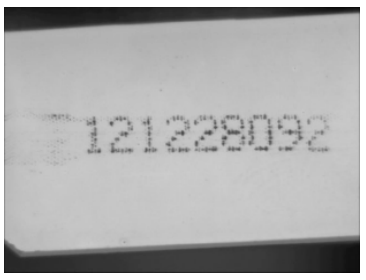

(d)

Fig. 2. Four online captured raw images For DMDC. (a) The raw image 1, (b) the raw image 2, (c) the raw image 3 and (d) the raw image 4 .

detection construction is shown in the Figs. 1(c) and $1(\mathrm{~d})$.

On the automatic blood smear producing line, the DMDCs used for identity authentication need to be printed online by stylus printer. Their postures captured by Charge-Coupled Device (CCD) may be protean due to the uncertainty of the working environments. Figure 2 shows four online captured raw images at different times. And Fig. 3 shows the zoom in for the dashed region in Fig. 2(a) in which the DMDC definitely consisted of multiple scattered small dots and some of the dots have been faded out. We can summarize some of the findings from Fig. 2: The postures of the DMDCs may be ever-changing in left or right deviation, up or down deviation and left or right incline, the contrasts of the DMDCs may be different; furthermore, the background noises of the DMDCs may be different either; besides, in these images we can also see some ruleless residues caused by ink ribbon online.
It is easy to find that the widths of the digits in the DMDCs are the same, except digit "1" which usually possesses half width of the others. It will arouse a special attention in DMDC location and digit segmentation. ${ }^{20,21}$

To eliminate or reduce the impacts on the DMDC identification, the special image processing strategy including image binarization with DMDC feature highlighting, DMDC location, and digit segmentation must be implemented comprehensively.

\subsection{Image binarization with an adjusted OTSU threshold and DMDC feature highlighting}

As we know, OTSU method, ${ }^{22,23}$ also known as maximum between-cluster variance method, is universally accepted for foreground segmentation. For a captured raw image $I(m, n)$ with a size of $M \times N$ pixels, if $T \in(0,255)$ is an assumed threshold, the

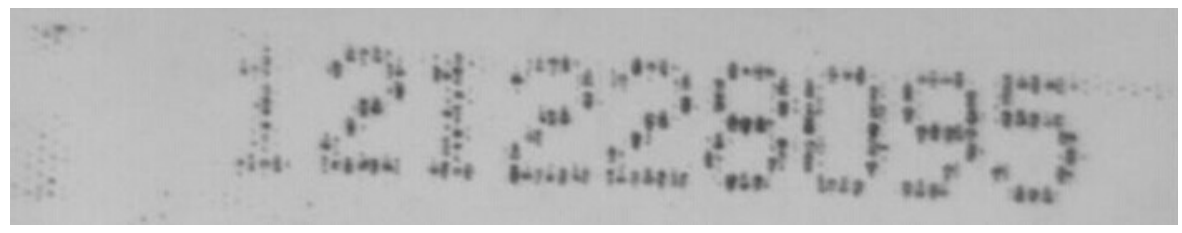

Fig. 3. Zoom in for the dashed region in Fig. 1(a). 
binarized image $I_{T}^{B}(m, n)$ for $I(m, n)$ is:

$$
\begin{aligned}
& I_{T}^{B}(m, n) \\
& \quad=\left\{\begin{array}{lll}
255 & \text { if } I(m, n) \geq T ; & m \in[0, M), n \in[0, N) \\
0 & \text { if } I(m, n)<T ; & m \in[0, M), n \in[0, N)
\end{array} .\right.
\end{aligned}
$$

The regions with 0 are segmented as the background and the others are segmented as the foreground, the foreground average gray $\bar{g}_{f}(T)$, the background average gray $\bar{g}_{b}(T)$ and the whole average gray $\bar{g}$ of the captured image $I(m, n)$ can be figured out respectively, so does the foreground proportion $\bar{p}_{f}(T)$. The variance between foreground and background is:

$$
\begin{aligned}
V(T)= & \bar{p}_{f}(T) \times\left(\bar{g}_{f}(T)-\bar{g}\right)^{2} \\
& +\left(1-\bar{p}_{f}(T)\right) \times\left(\bar{g}_{b}(T)-\bar{g}\right)^{2} .
\end{aligned}
$$

The optimal threshold value $T_{\text {OTSU }}$ in OTSU method is:

$$
T_{\mathrm{OTSU}}=\left.T\right|_{V(T)=\max \{V(T)\}} .
$$

OTSU method is a very popular image segmentation method for its auto-adaptivity.

Figure 4(a) is one of the captured images including DMDC. Figure 4(b) shows the result of the binarization of Fig. 4(a) with the OTSU threshold



(a)

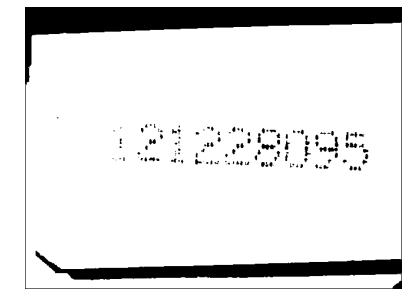

(b)

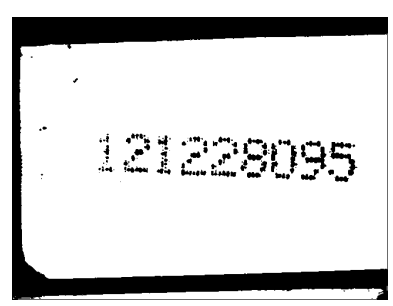

(c)

Fig. 4. Binary image with OTSU threshold and adjusted threshold respectively. (a) Captured raw image, (b) binarized by OTSU threshold and (c) binarized by adjusted threshold. in which the objective DMDC is under segmented. By analyzing the captured images, we can find that it is because there are many gray values overlapped between the DMDC region and the dark background region. And it is also found by many experimental results that there is an adjusted threshold $T_{\text {adj }}$ by which the DMDC can be segmented better, and the $T_{\text {adj }}$ is just offsetted $T_{\text {OTSU }}$ by a constant $T_{0}$ :

$$
T_{\text {adj }}=T_{\mathrm{OTSU}}+T_{0} .
$$

Figure $4(\mathrm{c})$ is the result of the binarization of Fig. 4(a) with the adjusted threshold which shows a better segmentation for DMDC.

Because the adjusted threshold is based on the OTSU threshold and $T_{0}$ is a constant determined by lots of experimental results, it is also auto-adaptive. Figure 5(a) shows an other captured image at different time with different illumination condition. Figure 5(b) shows almost the same well binarized result as Fig. 4(c) with the same auto-adaptive $T_{\text {adj }}$. In order to highlight the DMDC feature in the binarized image, three additional image processing steps must be implemented. Firstly, all white regions except the maximum rectangular white region must be filtered out. It is accomplished by maximum area filtering method as shown in Fig. 5(c). Secondly, the background should be exchanged for the foreground by image inverting to highlight the DMDC region of interest (ROI) as

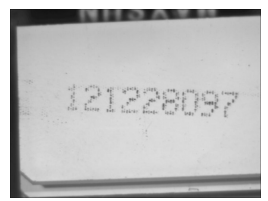

(a)

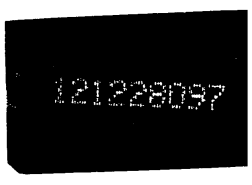

(d)

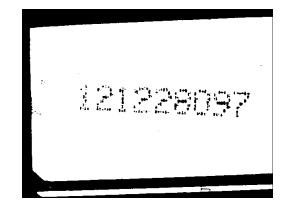

(b)

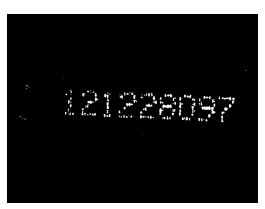

(e)

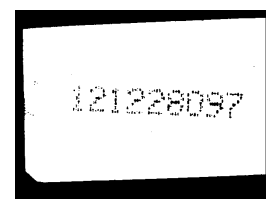

(c)



(f)
Fig. 5. Image processing procedure for DMDC feature highlighting. (a) Captured raw image, (b) binarized image by adjusted, (c) filtering out the white texts, (d) the invert image of (c), (e) filtering out white non-ROI and (f) highlighted DMDC by IMD. 
shown in Fig. 5(d). Thirdly, the other white nonROI surrounding the DMDC should be filtered out by a certain area threshold to eliminate the background noise as much as possible. The processing result is shown in Fig. 5(e). It is found that the DMDC feature is remarkable though there is still a little bit small noise caused by ruleless residues in Fig. 5(e). In order to further highlight the DMDC feature and benefit the DMDC identification, image morphological dilation (IMD) based a $5 \times 5$ circular template is applied. The result image of IMD is in Fig. 5(f), which performs well to highlight the DMDC feature.

\subsection{DMDC coarse location based on projection mapping}

As analyzed above, the DMDC location in the online captured image may not be fixed, and there may be up or down deviation in its position. Horizontal image projection mapping can summarize the pixels distribution of the foreground along the vertical direction. Figure 6(a) shows the result for Fig. 5(f) in which the largest nonzero continuous region represents the vertical position of the DMDC while the other smaller regions represent the noises and should be filtered out. Figure 6(b) is the filtering result in which the leftmost nonzero position shows the top of the DMDC and the rightmost

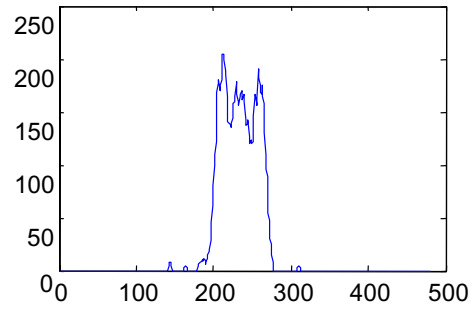

(a)

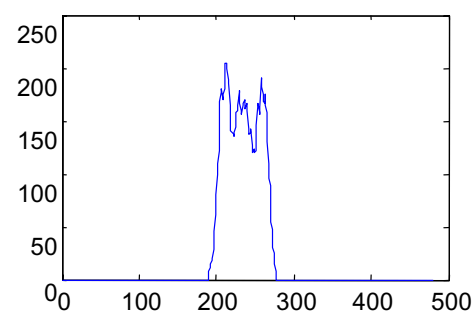

(b)

Fig. 6. Horizontal projection of Fig. 4(f) and filtering 0. (a) Horizontal projection curve and (b) after noises filtering out.



(a)

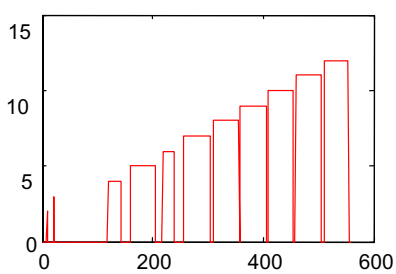

(c) (b)

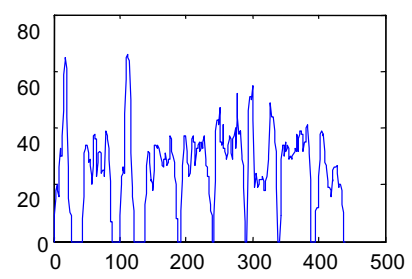

(d)

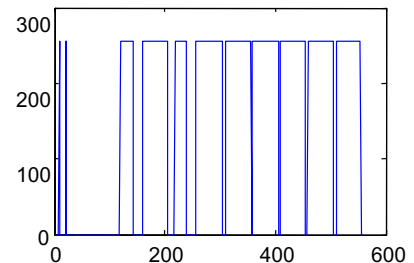

Fig. 7. Vertical projection mapping of Fig. 4(f) and filtering. (a) vertical projection curve, (b) binary projection curve, (c) region labeling and (d) after noises filtering out.

nonzero position shows the bottom of the DMDC roughly. There also may be left or right deviation in the position of the DMDC. Similarly vertical image projection mapping can summarize the pixels distribution of the foreground along the horizontal direction. Figure 7(a) shows the result for Fig. 5(f). Because the DMDC is composed of many dots and the length of DMDC is also an undetermined parameter, any filtering operation may lead to a loss of DMDC message. The leftmost nonzero position in Fig. 7(a) may show the left of the DMDC and the rightmost nonzero position may show the right of the DMDC roughly. In this way, the DMDC region can be cut out from Fig. 5(f) as shown in Fig. 8(a) which reveals some noises. In order to filter the noises and estimate the number of digits in DMDC, binarizing Fig. 7(a) with threshold of zero as shown in Fig. 7(b) and labeling the binary projection curve as shown in Fig. 7(c). The widths and symcenter positions of every labeled rectangle along the horizontal direction in Fig. 7(c) can be computed. Because the noises have narrower width and they are more far from the DMDC, the noises can be filtered out by narrower width filter and maximum between-cluster variance method as shown in Fig. 7(d). And the number of digits in the DMDC can also be figured out to be 10 by the width information from Fig. 7(c). Figure 8(b) shows the DMDC region after noises filtering out. But there is still a little bit right incline. The rotation 
X. Feng et al.

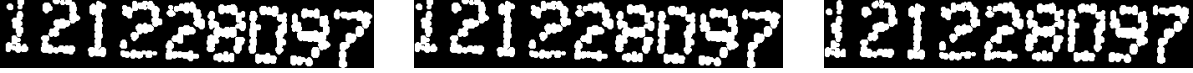

(a)

(b)

(c)

Fig. 8. Cut out DMDC image and its treatment. (a) Cut out DMDC image, (b) image after noise elimination, and (c) image after incline correction.

transformation method can adjust the DMDC to be a horizontal alignment as much as possible as shown in Fig. 8(c). Till this point, the DMDC has been located basically.

\subsection{DMDC fine location and its digits segmentation}

Again by projection mapping, binarization and labeling for the coarsely located DMDC image as shown in Fig. 8(d), the boundary of the DMDC can be determined by the four points with dash-circle marked as shown in Figs. 9(a) and 9(b), and the number of digits in DMDC can be affirmed exactly from Fig. 9(d). But the boundary determined in this way may not be the exact boundary of the DMDC. Observing the digits in the identified code, it is found that the digit " 1 " has the unique width half of that of the other digits as shown in Fig. 9(c). If the digit " 1 " is side-located in the DMDC, the corresponding boundary must be extended half of the width of digit "1", otherwise, the boundary of the DMDC do not need to be modified. Figure 9(a) shows the DMDC fine location while the digit " 1 " is left side located. The left boundary has been left

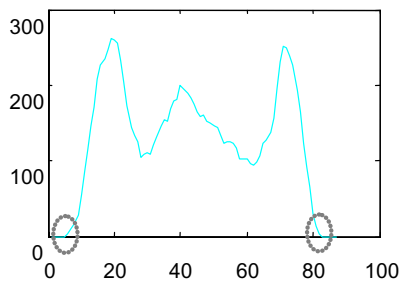

(a)



(b)

extended. Figure 10(b) shows the DMDC fine location while the digit " 1 " is both side located. The left and the right boundary have both been extended. Figure 10(c) shows the DMDC fine location while no digit " 1 " is side located. The boundary is just determined by the four points with dash-circle marked as shown in Figs. 9(a) and 9(b), has been left extended. The curves of the image after incline correction are shown in Fig. 8. By scanning the curve, the zero-points marked in the Figs. 9(a) and 9 (b) can be easily found, hence the precise boundary of the object area containing the code can be found. We can find that the width of the digit is equal to each other.

Because the DMDC images are captured online, the sizes of DMDCs at different captured images may be different. It may lead to wrong digits segmentation for the variable DMDC width and certainly lead to wrong DMDC identification. ${ }^{24}$ Different sizes of DMDCs must be normalized. The size of the fine located DMDC image as shown in Fig. 10 is $S_{w} \times S_{h}$, and the number of digits in the fine located DMDC is $N_{d}$ as affirmed above. The size of each individual digit to be identified is normalized as $W_{0} \times H_{0}$. The fine located DMDC image



(c)

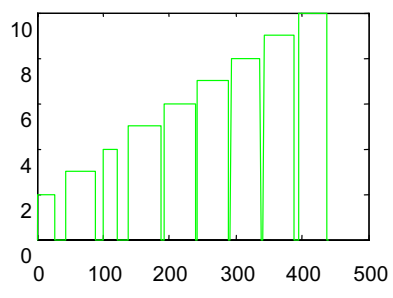

(d)

Fig. 9. Projection mapping curves and processing. (a) Horizontal projection mapping, (b) vertical projection mapping, (c) binary vertical projection mapping and (d) region labeling.



(a) (b) (c)

Fig. 10. DMDC fine location. (a) While digit "1" left side located, (b) while digit "1" both side located and (c) while no digit "1" side located. 


\section{7}

(a)

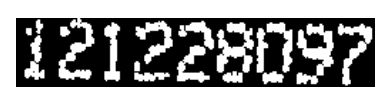

(b)

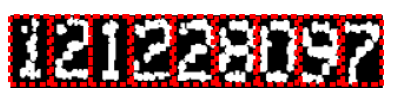

(c)

Fig. 11. Comparison between the images before and after normalization. (a) Image before normalization, (b) image after normalization and (c) digits segmentation.

can be normalized by zooming in or out from size of $S_{w} \times S_{h}$ to that of $W_{0} \times H_{0} \times N_{d}$ as shown in Fig. 11. As the input of the DMDC identification part, the individual digit image is required to be segmented from the DMDC. It can be easily accomplished with a fixed interval of $W_{0}$ in the normalized DMDC image as shown in Fig. 11(c).

\section{An Online DMDC Identification Method based on Heterological Theory}

Feature extraction is at the core of the DMDC identification. On ideal conditions, the segmented 10 digits " $0-9$ " have 10 different foreground pixel distributions and ten different sums of foreground pixels precisely, so they are very distinguishable by above features. Nevertheless, in actual cases, the segmented individual normalized digits " $0-9$ " are inevitable to be out of shape to various degrees. Figure 12 shows five typical segmented individual digits "9" at different time online. Their pixel distributions and sums of foreground pixels are not any longer the same and may be variable in a large range. The position of the digit and the thickness of the strokes are neither the same, and there may be some deficiencies in the digit. Fortunately, it can be solved by the robustness of subarea features. So we proposed an online DMDC identification method based on subarea features synthesization with heterological theory. We named the rectangle subarea of the individual digit image with determined position and size, just like the sub-region marked out in
Fig. 12 as $S_{n}$, where $n$ refers to the label in the rectangle. As the Fig. 12 shows, the subareas of the No. 1 and the No. 2 sub-regions are named as $S_{1}$ and $S_{2}$, respectively. It is obvious that $S_{1}$ and $S_{2}$ of all the digit "9" images are both small. That is $S_{1} \approx 0$ and $S_{2} \approx 0$. According to the experiments and observations, there must be some certain subarea features in common, whose position and size can be obtained by statistical approach, between all the images of each digit, and with which the digit image can be distinguished from other digits. For instance, seen in Fig. 13, no one digit but "9" can meet the criteria, $S_{1} \approx 0$ and $S_{2} \approx 0$. Thus, digit "9" can be distinguished.

In order to identify the digits with high accuracy and high speed, it is very important to get an appropriate number of subarea features for use. A feature selection method based on heterological principle is applied to aim at those discontinuous stroke subarea features with very small $S_{n}(\approx 0)$.

Heterological subarea feature concerns more about the subarea with background pixels than that with foreground pixels. Indeed, the subareas with foreground pixels are the components of the identified digit, but they are all lossy more or less in the online image capturing and image processing. Too many subarea features from these regions may lead to a too complex identification. If some unique subarea features can be selected from these regions for digits "0-9", they can be used as the auxiliary features. That is, synthesizing the heterological subarea features and these unique subarea features dynamically, the digit can be identified. For instance, digit "9" can be identified according to the

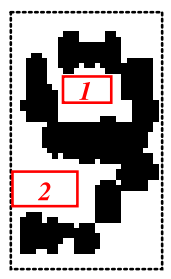

(a)

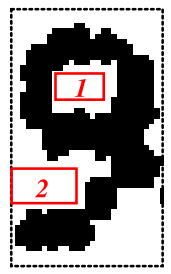

(b)

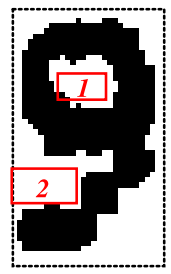

(c)

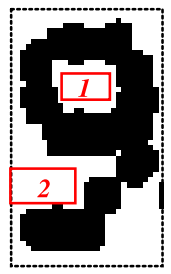

(d)

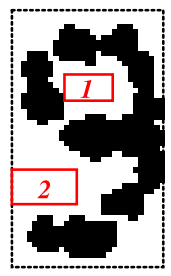

(e)

Fig. 12. Several "9" individual images segmented above. 


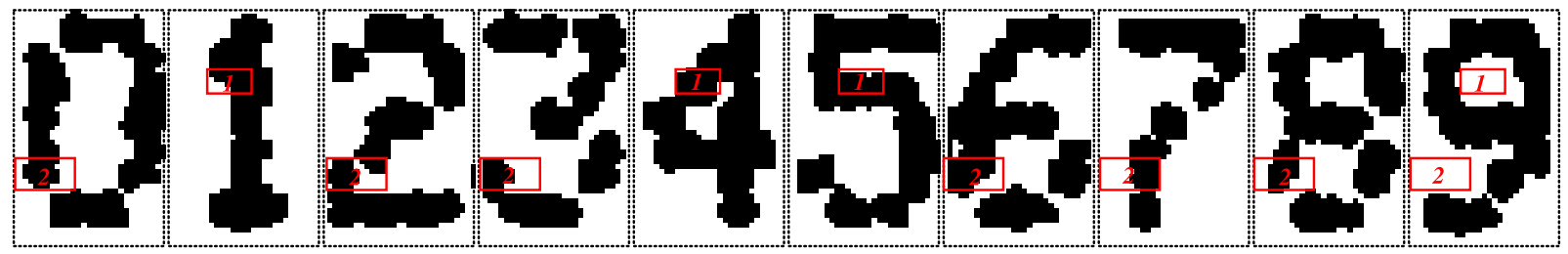

Fig. 13. Individual images with grids.

subarea features synthesization like this:

input digit "?"

$$
\begin{aligned}
& \Rightarrow \operatorname{if}\left\{\begin{array}{ll}
s_{1}=0 & (\text { "1" "4" "5" excluded }) \\
s_{2}=0 & (\text { "0" " } 2 " \text { " } 3 " \text { " } 6 " \text { " "7" "8" excluded })
\end{array}\right\} \\
& \Rightarrow \text { it must be digit " } 9 \text { ". }
\end{aligned}
$$

In this way, the distinct subarea features can guarantee the identification accuracy, and fewer subarea features can guarantee the identifying speed. With this method, the identification criterion for the other digits can be easily summarized.

\section{The Experiment Results and Analysis}

In order to verify its feasibility and validity of the proposed online identity authentication method for blood smear, it is successfully applied to an online blood smear automatic microscopic image detection system. Figure 14 shows parts of the automatic blood smear production system. After a robot completes smearing the patient's blood onto the glass slide to be a blood smear, the blood smear is transmitted by the conveyor through the stylus printer, and the DMDC is online printed by the print head as the patient's identifier for the blood smear. Then the blood smear is transported by another robot automatically to an online blood smear automatic microscopic image detection system as shown in Fig. 14(b). An XY scanning microscope stage scans the blood smear automatically, and the microscopic imaging CCD automatically captures the blood cell images, at the same time the DMDC CCD captures the DMDC image online to acquire the patient's identity authentication of the blood smear. The correct DMDC identification can guarantee that the blood cell microscopic image detection result corresponds to the right patient and a wrong one will lead to a serious medical accident.

More than 1000 blood smears have been produced automatically with blood smear production system and transported by a robot automatically to the online blood smear automatic microscopic image detection system for DMDC identification test and microscopic image detection online. It is found that all the online DMDC identification results reveal the correct DMDC and the average identification time is less than half a second. It shows that the proposed method can meet the

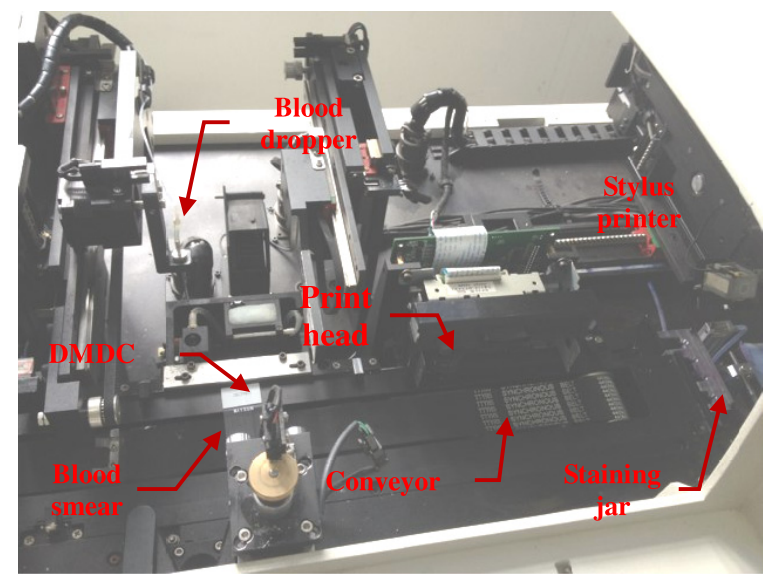

(a)

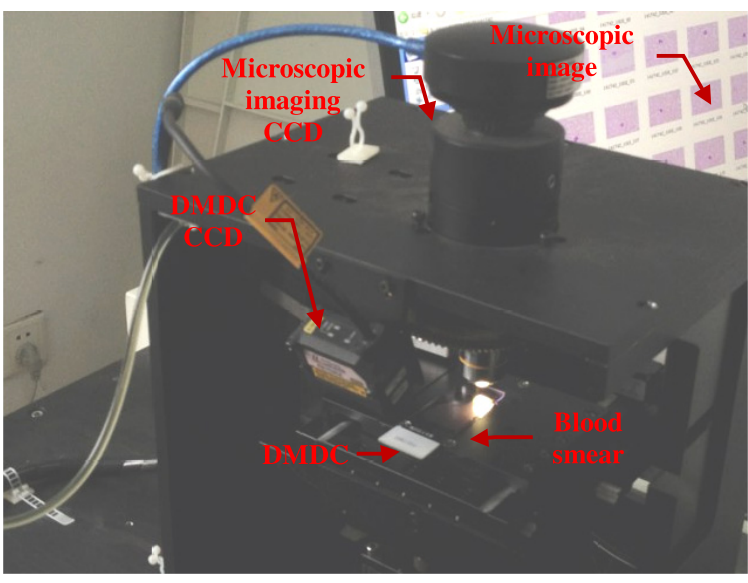

(b)

Fig. 14. Experimental system. (a) Part of the blood smear production system and (b) online blood smear microscopic image detection system. 


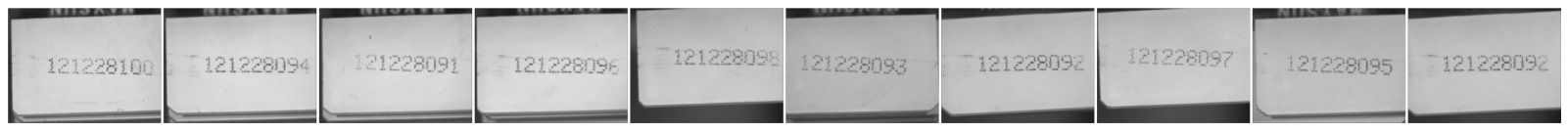

(a)

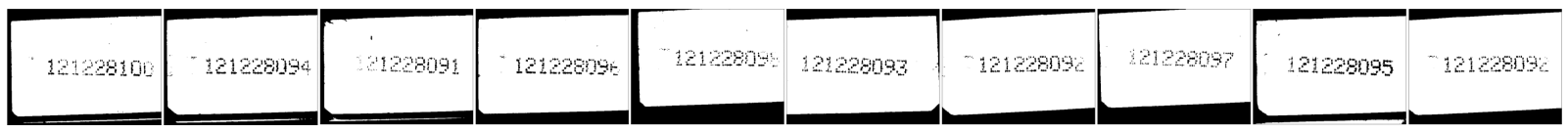

(b)



(c)



(d)

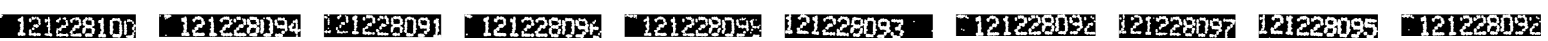

(e)

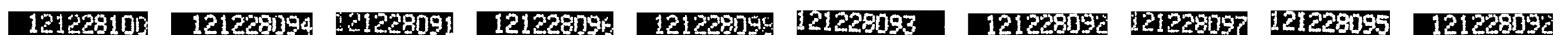

(f)

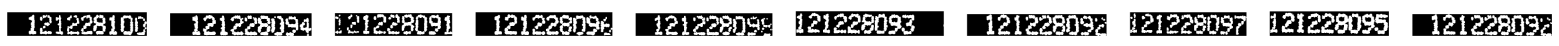

(g)

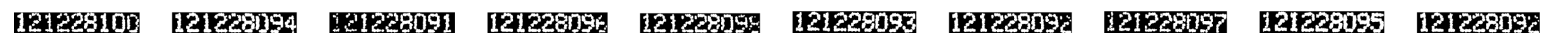

(h)



(i)

$121228100,121228094,121228091,121228096,121228098,121228093,121228092,121228097,121228095,121228092$

(j)

Fig. 15. Blood smear identity authentication procedure and identification results. (a) Captured DMDC images, (b) binarized DMDC images, (c) highlight DMDC images by filtering out background, (d) further highlight DMDC Images by morphological dilation, (e) cut out DMDC images, (f) images by filtering out noise, (g) images after incline correction, (h) DMDC by precise location, (i) normalized DMDC and (j) the identification results.

requirement for online DMDC identification with high accuracy.

Here the DMDC identification processing procedure and identification results for 10 blood smears in Fig. 15 are shown. There are 10 typical captured DMDC images in Fig. 15(a). They are all different in their location, their illumination, their background or their posture. Their binarized images by 
the adjusted OTSU threshold are shown in Fig. 15(b) which reveal good DMDC trait. By filtering out the background, the DMDC images can be highlighted as shown in Fig. 15(c). By IMD operation, the DMDC images can be highlighted further as shown in Fig. 15(d). By horizontal and vertical projection mapping, the DMDC can be cut out as shown in Fig. 15(e) though there may be a little bit noises in some of these images. By vertical projection curve binarization, labeling and maximum between-cluster variance method, the noises can be filtered out successfully as shown in Fig. 15(f) though there may be a little bit incline in some of these images. By rotation transformation method, the DMDC can be a horizontal alignment as shown in Fig. 15(g). In this way, the coarse location of DMDC has been accomplished. By horizontal and vertical projection mapping again and considering the unique narrower width of digit " 1 ", the fine location of DMDC can be achieved as shown in Fig. 15(h). By DMDC normalization as shown in Fig. 15(i), each digit in the corresponding DMDC can be segmented and identified accurately. Figure 15(j) shows the DMDC identification results by the proposed DMDC identification method.

We can find that the identification results successfully match the corresponding tube barcode. The experimental results show the feasibility and validity of the proposed method for online DMDC identification.

Considering the requirement for high speed, we analyzed the computation cost of the identification process. The time costs of the 10 samples in Fig. 15(a) are shown in Table 1.

Table 1. Computation cost of the identification processes.

\begin{tabular}{cccc}
\hline & \multicolumn{3}{c}{ Time cost (s) } \\
\cline { 2 - 4 } Samples & Feature extraction & Digit identification & Sum \\
\hline 1 & 0.348644 & 0.076270 & 0.424914 \\
2 & 0.312556 & 0.072532 & 0.385088 \\
3 & 0.351604 & 0.078594 & 0.430198 \\
4 & 0.349498 & 0.077702 & 0.427200 \\
5 & 0.286946 & 0.074767 & 0.361713 \\
6 & 0.367659 & 0.074845 & 0.442504 \\
7 & 0.334323 & 0.079730 & 0.414053 \\
8 & 0.323466 & 0.070563 & 0.394029 \\
9 & 0.332413 & 0.083566 & 0.415979 \\
10 & 0.355424 & 0.075941 & 0.431365 \\
Average & 0.336253 & 0.076451 & 0.412704 \\
\hline
\end{tabular}

In Tabel 1, the maximum and the average of the time costs are both found to be less than half a second. And the amounts of experiments show the same situation that the identification times are all less than half a second, which can meet the requirement of the online system.

\section{Conclusion}

Overall considering the particularities of DMDC and the complexities of online application environment, an online identity authentication method for blood smear based on subarea features synthesization with heterological theory is proposed. It can locate finely DMDC and identify DMDC with different number of digits automatically. Less than half second identity authentication time shows that the proposed method can meet the requirement for online identity authentication for blood smear with high accuracy. It has been successfully applied in the patient identification of online blood smear automatic microscopic image detection. The experimental results verified its feasibility and validity.

\section{Acknowledgments}

This work was supported by the 863 National Plan Foundation of China under Grant No. 2007AA01Z333 and Special Grand National Project of China under Grant No. 2009ZX02204-008.

\section{References}

1. F.-G. Debray, C. Lefebvre, S. Colinet, K. Segers, R. Stevens, "Free sialic acid storage disease mimicking cerebral palsy and revealed by blood smear examination," J. Pediatrics 158(1): 165-165.e1 (2011).

2. J. F. Lesesve, "Cryoglobulin deposits on a blood smear," Blood, 120(9): 1764-1764 (2012).

3. H. Park, D. Shafer, "Disseminated histoplasmosis in peripheral blood smear," Blood, 123(10): 1445-1445 (2014).

4. B. J. Bain, "Diagnosis from the blood smear," New Eng. J. Med. 353(5): 498-507 (2005).

5. I. Y. Takihi, A. F. Sandes, "Killers on the road: Klebsiella and pseudomonas bacteremia detected on peripheral blood smear," Blood, 122(11): 1851 (2013).

6. R. Radha, R. R. Aparna, Digit recognition using hybrid classifier, 2014 World Congress on Computing and Communication Technologies, pp. 34-38, February 2014. 
7. V. N. More, P. P. Rege, Devanagari handwritten numeral identification based on Zernike moments, TENCON 2008 - 2008 IEEE Region 10 Conf. pp. 1-6, November 2005.

8. O. D. Trier, A. K. Jain, T. Taxt, "Feature extraction methods for character recognition-A survey," Pattern Recognit. 29(4): 641-662 (1996).

9. X. Wang, X. Ding, C. Liu, "Gabor filters-based feature extraction for character recognition," Pattern Recognit. 38(3): 369-379 (2005).

10. D. D. Gaurav, R. Ramesh, "A feature extraction technique based on character geometry for character recognition," Comput. Vis. Pattern Recognit., arXiv:1202-3884.

11. H. Grailu, M. Lotfizad, H. Sadoghi-Yazdi, "1-D chaincode pattern matching for compression of Bilevel printed farsi and arabic textual images," Image Vis. Comput. 27(10): 1615-1625 (2009).

12. C.-L. Liu, K. Nakashima, H. Sako, H. Fujisawa, "Handwritten digit recognition: Investigation of normalization and feature extraction techniques," Pattern Recognit. 37(2): 265-279 (2004).

13. B. V. Dhandra, R. G. Benne, M. Hangarge, "Handwritten Kannada numeral recognition based on structural features", Int. Conf. Computational Intelligence and Multimedia Applications, Vol. 2, pp. 224-228, December 2007.

14. J.-J. Park, T.-W. Kim, K.-M. Kim, "Handwritten numeral recognition using composite features and SVM Classifier," J. Korea Inst. Maritime Information Commun. Sci. 14(12): 2761-2768 (2010).

15. G. Y. Chen, T. D. Bui, A. Krzyzak, "Contour-based handwritten numeral recognition using multiwavelets and neural networks", Pattern Recogn. 36(7): 15971604 (2003).

16. L. Yang, C. Y. Suen, T. D. Bui, P. Zhang, "Discrimination of similar handwritten numerals based on invariant curvature features", Pattern Recogn. 38(7): 947-963 (2005).

17. I. A. Lawal, R. E. Abdel-Aal, S. A. Mahmoud, "Recognition of Handwritten Arabic (Indian) numerals using freeman's chain codes and abductive network classifiers," 2010 Int. Conf. Pattern Recognition pp. 1884-1887, August 2010.

18. R. Radha, R. R. Aparna, "Review of OCR techniques used in automatic mail sorting of postal envelopes," Signal Image Process. 4(5): 45-60 (2013).

19. M. T. Parvez, S. A. Mahmoud, "Offline arabic Handwritten text recognition: A survey," $A C M$ Computing Surveys, Summer, 45(2): 23-35 (2013).

20. N. R. Pal, S. K. Pal, "A review on image, segmentation techniques", Pattern Recognit. 26: $1277-$ 1294 (1993).

21. A. Sedighi, M. Vafadust, "A new and robust method for character segmentation and recognition in license plate images," Expert Syst. Appl. 38(11): 13497-13504 (2011).

22. P. J. Herrera, G. Pajares, M. Guijarro, "A segmentation method using Otsu and fuzzy k-Means for stereovision matching in hemispherical images from forest environments," Appl. Soft Comput. J. 11(8): 4738-4747 (2011).

23. N. Otsu, "A threshold selection method from greylevel histograms," IEEE Trans. Syst. Man Cybern., 9(1): 62-66 (1979). 